\title{
Entropy in quality systems
}

\author{
Stanley D. Rasberry
}

Published online: 10 December 2008

(C) Springer-Verlag 2008

In nature all systems are subject to the laws of thermodynamics. This is true for the largest star to the smallest living cell. No part of the law is escaped by any entity. The second law, that of entropy, especially is not escaped. For the cell the effects of degradation and death are seen over short periods of time, while the star may evolve over five billion years or more. Nevertheless, without the insertion of energy, even stars decay and die.

In simplest terms the second law of thermodynamics states that, between two bodies, heat will flow only from the warmer to the cooler. Any reversal of the process can be obtained only at a cost of energy equal to or greater than the amount of heat moved from the cooler body to the warmer body. In a practical way, we see this in our homes in wintertime. If no energy is expended, heat will be transferred from our home to the cooler outdoors until both reach the same temperature. To prevent that we may use an electric "heat pump" which extracts heat from colder air and moves it "uphill" to our home's warmer interior. The heat pump requires energy in an amount greater (because its efficiency is not perfect) than the quantity of heat moved. We are reminded of this in dramatic fashion at the moment we receive a winter-month electric bill.

Physical chemists will recall that entropy is reduced when water is frozen by applying work through a refrigerator to extract heat from the water. When the energy content of the water is reduced the entropy is also reduced, but the physical order of the molecules is increased with the formation of highly ordered ice crystals. If the ice is allowed to melt it becomes a disordered liquid and gains entropy. The natural trend, without exertion of work, is

S. D. Rasberry $(\bowtie)$

1255 Glebe Road, Lottsburg, VA 22511, USA

e-mail: rasberry@rivnet.net toward disorder and increase in entropy. The relationship between increase in disorder and increase in entropy has been noted by specialists in information science and applied in their field. Likewise social scientists have begun to apply the relationship in the analysis of breakdown in the order of complex social systems.

The conceptual issue for those who study quality systems is: does entropy have meaning in the context of procedural systems constructed solely for the purpose of governing operation of a specific process? I would argue that entropy does apply to such systems in a way that can be described at least qualitatively. There is no question that the specific process being controlled is fully subject to the laws of thermodynamics. The new question is whether quality systems are so abstract that they escape thermodynamic principles.

The argument in favor of accepting the qualitative role of entropy in such systems rests in the reality that they are neither created nor maintained without the input of human energy beyond mere application of intellect. They are not purely theoretical. The difficulty in advancing from qualitative to quantitative understanding stems from the absence of any metrology for "human work". Human work is often described in terms of man hours and man years, but there is no physical definition of these quantities. It is generally accepted that one worker may accomplish an objective in less time than needed by a different person, leading to nonequivalence of their respective man hours. Developing a quantitative understanding is a subject for future study, or perhaps it will never be possible.

That said, and if we accept the qualitative aspect, how does it work? One explanation could be as human work goes into the development and implementation of a quality system it becomes an orderly system of defined actions and thus is reduced in entropy by the degree of that ordering. 
After the quality system is in place and with the passage of time, the original order is not perfectly maintained. There may be several reasons for this, but common ones are: substitution of less trained for better trained personnel; unforeseen changes in environment around the controlled processes; and, variation in incoming materials.

Entropy is increased as the order decreases. Likewise there will be an unfortunate increase in variation of the product or service, and thereby a reduction in quality. Only with new input of work hours can personnel be trained and retrained, or the system adapted to meet new conditions.
If these things are done the entropy can be reduced and decay forestalled.

Thus, a quality system left alone may become useless due to increase in entropy.

The only antidote is to apply sufficient work to the system to retard the increase of entropy.

Stanley D. Rasberry

Director of Measurement Services (Retired)

National Institute of Standards and Technology (NIST) 